\title{
A survey of major trauma centre staffing in England
}

\author{
Jan 0 Jansen, ${ }^{1,2}$ J J Morrison, ${ }^{3,4}$ N R M Tai, ${ }^{4,5}$ M J Midwinter ${ }^{4,6}$
}

${ }^{1}$ Departments of Surgery and Intensive Care Medicine, Aberdeen Royal Infirmary, Aberdeen, UK

${ }^{2}$ Health Services Research Unit, University of Aberdeen,

Aberdeen, UK

${ }^{3}$ Academic Unit of Surgery, Glasgow Royal Infirmary, Glasgow, UK

${ }^{4}$ Academic Department of Military Surgery and Trauma, Royal Centre for Defence Medicine, Birmingham, UK ${ }^{5}$ Trauma Unit, Royal London Hospital, London, UK ${ }^{6}$ National Institute of Health Research, Surgical Reconstruction and Microbiology Research Centre, Queen Elizabeth Hospital, Birmingham, UK

\section{Correspondence to} Jan 0 Jansen, Departments of Surgery and Intensive Care Medicine, Ward 505, Aberdeen Royal Infirmary, Foresterhill, Aberdeen AB25 2ZN, UK; jan.jansen@abdn.ac.uk

Received 20 August 2014 Revised 12 November 2014 Accepted 14 December 2014 Published Online First 2 February 2015

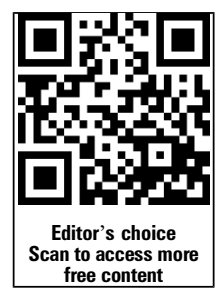

CrossMark

To cite: Jansen JO, Morrison JJ, Tai NRM, et al. J R Army Med Corps 2015;161:341-344.

\section{ABSTRACT}

Introduction Trauma care delivery in England has been transformed by the development of trauma networks, and the designation of trauma centres. A specialist trauma service is a key component of such centres. The aim of this survey was to determine to which extent, and how, the new major trauma centres (MTCs) have been able to implement such services.

Methods Electronic questionnaire survey of MTCs in England.

Results All 22 MTCs submitted responses. Thirteen centres have a dedicated major trauma service or trauma surgery service, and a further four are currently developing such a service. In 7 of these 17 centres, the service is or will be provided by orthopaedic surgeons, in 2 by emergency medicine departments, in another 2 by general or vascular surgeons, and in 6 by a multidisciplinary group of consultants.

Discussion A large proportion of MTCs still do not have a dedicated major trauma service. Furthermore, the models which are emerging differ from other countries. The relative lack of involvement of surgeons in MTC trauma service provision is particularly noteworthy, and a potential concern. The impact of these different models of service delivery is not known, and warrants further study.

\section{INTRODUCTION}

The past 5 years have seen a remarkable transformation of the delivery of major trauma care in England. Following many years of neglect ${ }^{1}$ and poor outcomes, ${ }^{2}{ }^{3}$ a national network of regional trauma systems has now been established. The highest echelons of care are formed by major trauma centres (MTCs), of which there are 22 across England. The biggest trauma network is in London, which comprises four MTCs, and this trauma system has already had a noticeable impact on outcomes. ${ }^{45}$

However, trauma systems and MTCs, are still at a nascent stage. A key area for development is the delivery of specialist trauma care following the initial assessment and management in the emergency department. ${ }^{16}$ This phase requires clinical leadership and ownership, as well as cross-specialty knowledge. The concept of a dedicated, surgically delivered service is central to the North American model of trauma care, and probably accounts-at least in part-for the improved outcomes in tertiary centres. ${ }^{7}$

The need for a similar subspecialty in the UK has been recognised for some time, ${ }^{16}$ and forms part of the Department of Health's key recommendations on the implementation of trauma networks. ${ }^{8}$ The key functions of such a service are given in Figure 1. Current guidance similarly calls for consultant-led initial assessment, and the colocation

\section{Key messages}

Trauma systems and trauma centres save lives and improve functional outcomes.

- These improvements are, at least in part, attributable to having dedicated major trauma services.

- In most health services with regionalised trauma care, major trauma services are provided by general surgeons.

- Some major trauma centres in the UK still do not have a major trauma service.

- Where available, the staffing of these services varies considerably.

- The impact of these different models of service delivery is not known, and requires further study.

of severely injured patients on dedicated wards. ${ }^{8}$ At present, it is not known to what extent MTCs in the UK have been able to implement this guidance. We therefore conducted a national survey of MTCs, to establish current staffing patterns.

\section{MATERIALS AND METHODS}

This is a questionnaire survey of major trauma centre clinical leads, or individuals with similar knowledge of the network, in England (see Acknowledgements section). The questionnaire comprised eight questions (Figure 2), and was administered electronically, using SurveyMonkey. Invitations were sent by email, containing a unique code, to enable the tracking of responses. Non-respondents were sent two further invitations by email. The results are presented as raw numbers.

\section{RESULTS}

Replies were received from all 22 adult MTCs (Figure 3). One of the 22 centres reports an annual case volumes of less than 240 severely injured patients per year, which is below the most conservative threshold shown to effect a change in mortality. Eleven centres report an annual volume of 241-400 severely injured patients per year, and eight MTCs report an annual case volume in excess of 400 severely injured patients per year (Figure 4).

All 22 centres have a trauma call procedure, but in one these, the response is not consultant-led. Only 13 centres have a dedicated major trauma service or trauma surgery service, although a further four are currently developing such a service (Figure 5). In 7 of these 17 centres, the service is or will be provided by orthopaedic surgeons, in 2 by emergency medicine departments, in another 2 


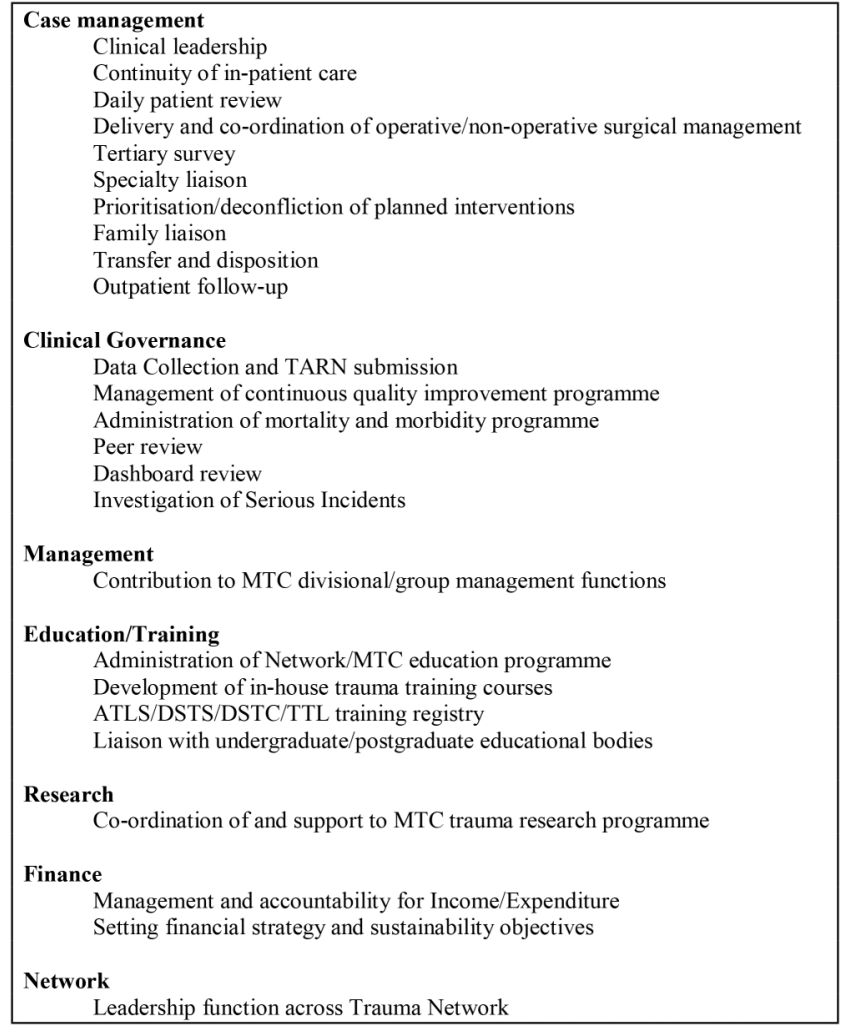

Figure 1 Key functions of a major trauma centre trauma service.

by general or vascular surgeons, and in 6 by a multidisciplinary group of consultants (Figure 6). Thirteen of the 21 centres have a dedicated ward for trauma patients.

\section{DISCUSSION}

This is the first study to examine the maturation of staffing structures in English MTCs. Almost all of the responding MTCs report an annual volume of more than 240 severely injured patients, which is deemed the minimum case volume required for a trauma centre to effect a change in mortality, ${ }^{8} 9$ although it is acknowledged that there is also evidence that the number may be as high as 400 severely injured patients per year, or even 650 severely injured patients per year. ${ }^{10}$ All of the MTCs have a trauma call procedure, although in one the response is not led by a consultant. This compares favourably with a survey

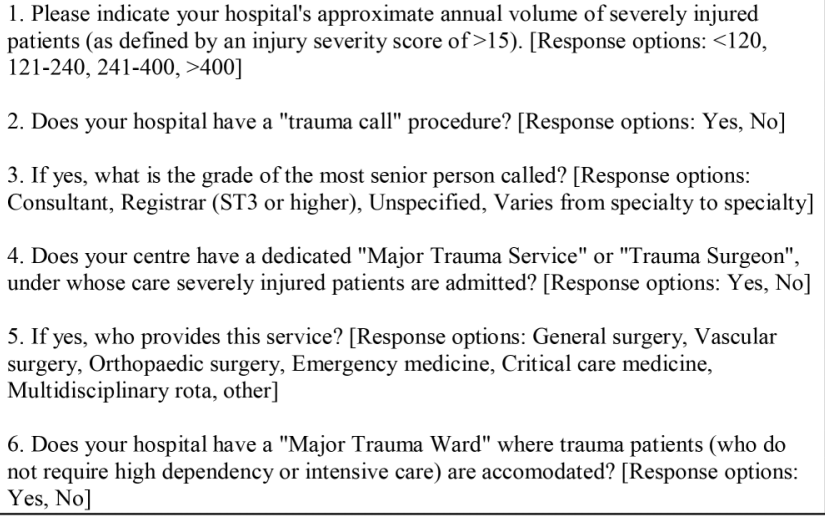

Figure 2 Questionnaire.

\begin{tabular}{|l|}
\hline Addenbrooke's Hospital, Cambridge \\
Frenchay Hospital, Bristol \\
James Cook University Hospital, Middlesborough \\
John Radcliffe Hospital, Oxford \\
Leeds General Infirmary \\
Queens Medical Centre, Nottingham \\
Royal London Hospital \\
Royal Victoria Infirmary, Newcastle \\
St Mary's Hospital, London \\
St George's Hospital, London \\
Southampton General Hospital \\
Derriford Hospital, Plymouth \\
King's College Hospital, London \\
Hull Royal Infirmary \\
Northern General Hospital, Sheffield \\
Queen Elizabeth Hospital, Birmingham \\
Royal Preston Hospital \\
Royal Susses County Hospital, Brighton \\
University Hospital, Coventry \\
University Hospital of North Staffordshire, Stoke on Trent \\
Manchester Collaborative MTC \\
Liverpool Collaborative MTC \\
\hline
\end{tabular}

Figure 3 Participating major trauma centres.

performed in 2008, although this previous study examined all hospitals, rather than MTCs in particular. ${ }^{11}$

The importance of a dedicated service to care for trauma patients-following their initial assessment and treatment-is increasingly recognised. It is therefore concerning that only just over half of the centres have instituted such a service, although it should be noted that several others are planning to develop a trauma service. Providing a consultant-delivered major trauma service has cost implications, and is difficult to justify when the volume of severely injured patients is low. It is conceivable that a further rationalisation of services may have to be considered in areas where hospitals are unable to provide such a service, although there are other considerations-such as accessibilitywhich need to be taken into account.

It is also noteworthy that the service models which are emerging differ from those in other parts of the world. At present,

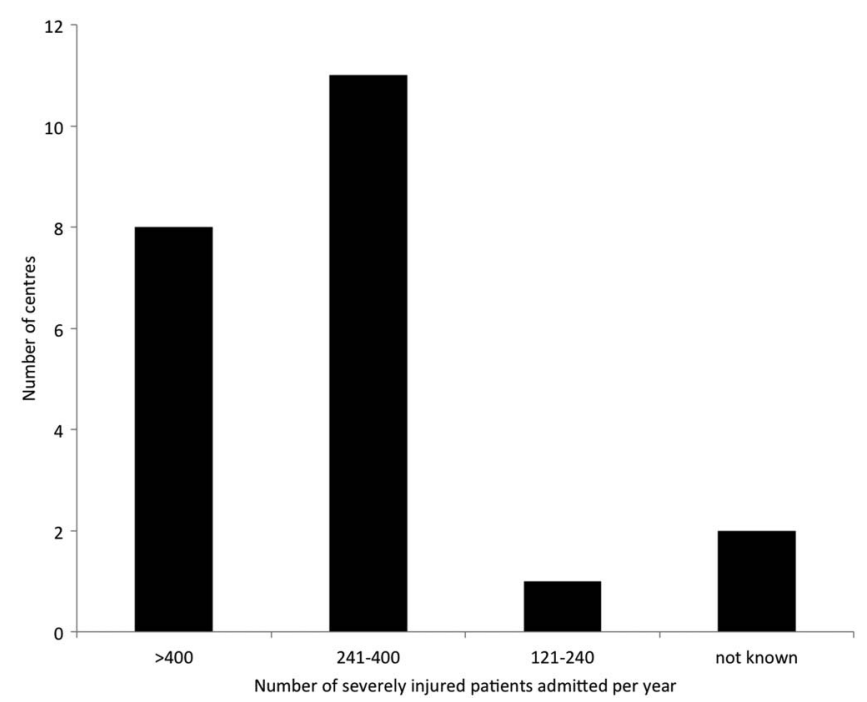

Figure 4 Major trauma centre volume. 


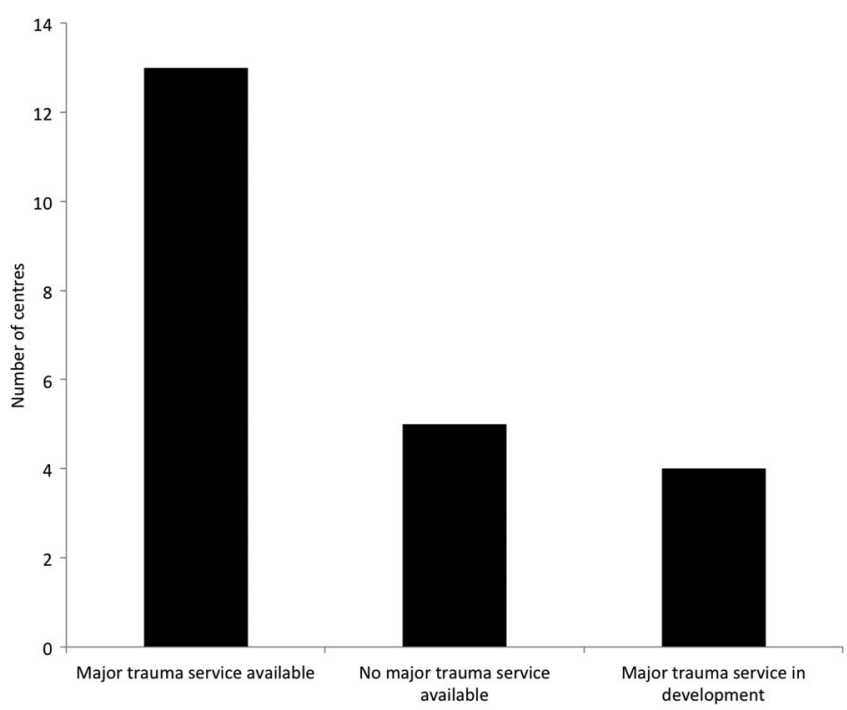

Figure 5 Availability of a major trauma service.

only two of the MTCs, both in London, have a trauma service comparable to that of a US trauma centre, comprising surgeons with a general/vascular surgical background. Taking into consideration the additional MTCs with orthopaedic trauma service provision, only 9 of England's 22 MTCs have developed a template that places surgeons at the centre of on-going care for the major trauma patient. The extent to which such models of service have been the result of methodical design, based on local injury patterns and patient need, was not assessed in our survey. We also did not assess the essential competencies required to deliver trauma care, although it is acknowledged that these will be delivered in part by engagement with other specialties.

Different MTCs will have been led by trauma champions from a variety of clinical backgrounds. Emergency medicine physicians in the UK have made major and sustained contributions to acute trauma care, and have been vigorous in developing the emerging specialty of prehospital care. Similarly, other specialties represented on the multidisciplinary rotas-in particular, anaesthesia and critical care medicine-often have considerable experience of managing abnormal physiology,

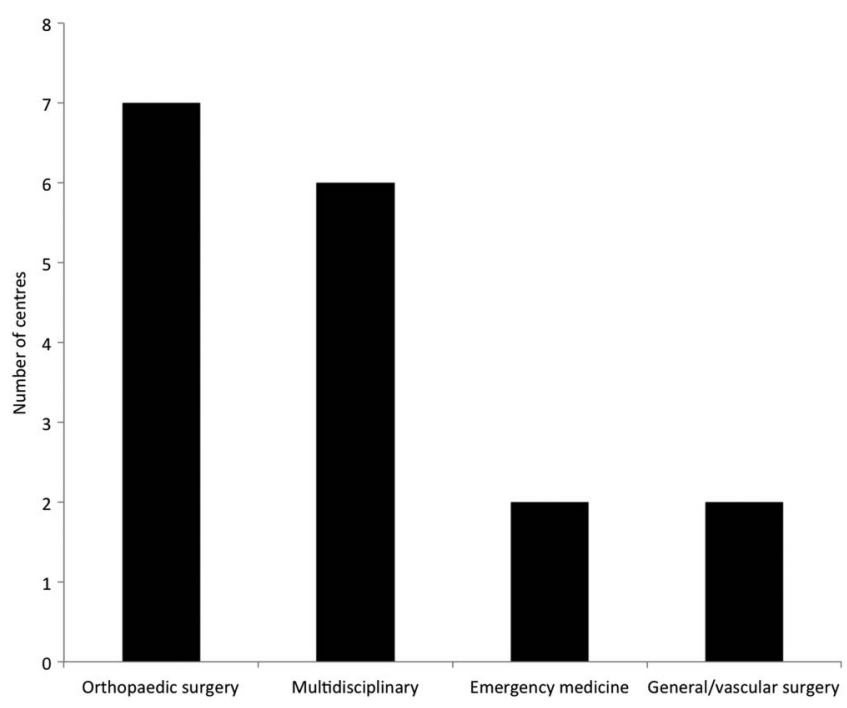

Figure 6 Specialties providing major trauma service (at 17 centres). although neither of these specialties have traditionally had continuity of care responsibility. However, trauma-trained surgeons offer advantages to the on-going care of the injured, as continuity of named consultant care is fundamental to British surgical practice, and can thus assure the patient pathway from admission through to discharge and beyond. This is particularly important when, for example, non-operative management is considered; where continual repeat assessment and evaluation is required to change to an interventional approach should the non-operative strategy fail. Furthermore, many of the functions of a trauma service, such as performance improvement, planning discharge arrangements, and ensuring follow-up, are core functions of modern surgical practice.

In addition to addressing the ongoing care of patients who have suffered major trauma, staffing models should also consider the ability of a trauma service to manage the most common preventable cause of death after injury, which is non-compressible torso haemorrhage. The number of patients who present with exsanguinating haemorrhage is small, but decisive and proactive management can profoundly affect outcome. Trauma-trained general surgeons are best placed to diagnose and treat these patients, whether by surgery or through collaboration with interventional radiology services. However, the ability to justify a trauma surgical service-as opposed to a service led by another specialty-partly depends on the volume of such patients, which is related both to overall volume, and to regional case mix. This situation may be less problematic in areas of the UK where penetrating or highenergy blunt trauma is infrequent. However, in those high-volume MTCs catering to populations where surgical intervention is common, this variation in practice raises questions. Furthermore, there are presently very few trauma-trained general surgeons in the UK, and as trauma is not recognised as a general surgical subspecialty, there are no recognised training programmes to equip surgeons who would like to specialise in trauma care with the competencies identified in the most recent 2013 edition of the Intercollegiate Surgical Curriculum. ${ }^{12}$ In order to rectify the training and experience gap for trauma surgery, there needs to be leadership from the profession to develop the opportunities to gain the necessary competencies and skills.

\section{CONCLUSION}

In summary, this survey shows that major trauma services in England are continuing to mature, although there are still MTCs without a dedicated trauma service. Trauma services are developing in a different direction compared to North America. The process by which MTCs chose their model of major trauma service delivery, and the consequent impact of these different models of service delivery, is not known, and warrants further study.

Acknowledgements The authors would like to thank Rod Mackenzie (Cambridge), David Lockey (Bristol), Adam Kempster (Middlesborough), John McMaster (Oxford), Kate Prior (London), Ray Monkhouse (Leeds), Adam Brooks (Nottingham), Wayne Sapsford (London), Reuben Saharia (Newcastle), Chris Aylwin (London), Heather Jarman (London), Andy Eynon (Southampton), Tony Kehoe (Plymouth), Ben Rayner (Hull), Stuart Reid (Sheffield), Andy Curran (Preston), lain MacFadyen (Brighton), James Davidson (Coventry), Simon Davies (Stoke), Beverley Thompson (Manchester), Badri Narayan (Liverpool) for completing the survey on behalf of their hospitals. We would also like to thank Conor Thompson (University of Aberdeen) for his help in administering the survey.

Contributors JOJ planned the survey, designed the questionnaire, analysed the responses, and drafted the manuscript. JJM, NRMT and MJM assisted with the interpretation of the findings and revised the manuscript. JOJ is the guarantor.

Funding None. 
Disclaimer The Health Services Research Unit receives funding from the Chief Scientist Office of the Scottish Government Health and Social Care Directorates. The opinions expressed in this article are those of the authors alone.

Competing interests None.

Provenance and peer review Not commissioned; externally peer reviewed.

\section{REFERENCES}

1 Tai NRM, Ryan JM, Brooks AJ. The neglect of trauma surgery. Br Med J 2006;332:805

2 Findlay G, Martin IC, Carter S, et al. Trauma: who cares? London: NCEPOD, 2007.

3 Royal College of Surgeons of England, British Orthopaedic Association. Better care for the severely injured. London: RCSE, 2003.

4 Davenport RA, Tai N, West A, et al. A major trauma centre is a specialty hospital not a hospital of specialties. Br J Surg 2010;97:109-17.
5 NHS England. Independent review of major trauma networks reveals increase in patient survival rates. http://www.england.nhs.uk/2013/06/25/incr-pati-survi-rts/ (accessed 19 Nov 2013).

6 Jansen JO, Tai NRM, Midwinter J. Planning trauma care services in the UK. Br Med J 2013;346:f738.

7 MacKenzie EJ, Rivara FP, Jurkovich GJ, et al. A national evaluation of the effect of trauma-center care on mortality. N Engl J Med 2006;354:366-78.

8 NHS Clinical Advisory Groups. Regional networks for major trauma. 2010.

9 American College of Surgeons Committee on Trauma. Resources for optimal care of the injured patient: 2006. Chicago, 2006.

10 Nathens $A B$, Jurkovich GJ, Maier RV, et al. Relationship between trauma center volume and outcomes. JAMA 2001;285:1164.

11 Andrews JM, Dickson EJ, Loudon MA, et al. Protocol-driven trauma resuscitation: survey of UK practice. Emerg Med J 2009;26:864-5.

12 Intercollegiate surgical curriculum programme. https://www.iscp.ac.uk/surgical/ SpecialtySyllabus.aspx?spec_id=104 (accessed 4 Mar 2014). 